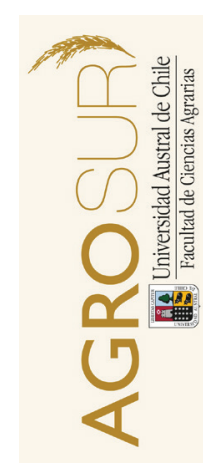

\title{
Desarrollo y evaluación de una unidad sembradora de maíz (Zea mays L.) para tracción animal o humana
}

\author{
Development and evaluation of a corn (Zea mays L.) \\ seeding unit for animal or human traction
}

A RT I C LE INFO

Keywords:

Intermediate technology

Mechanization

Planting

Small farmers

Zea mays

Original Research Article,

Agriculture Engineering

and Energy Management

*Corresponding author:

Ian Homer

E-mail address:

ihomer@uchile.c]

\author{
Homer, $\mathbf{I}^{a *}$, Villa, ${ }^{a}$
}

a Universidad de Chile, Facultad Ciencias Agronómicas Departamento Ingeniería y Suelos. Santa Rosa 11315, La Pintana, Santiago, Chile.

\section{A B S T R A C T}

Mechanization is generally difficult for small farmers since it is either not accessible due to cost or it does not adapt to their necessities. Much effort has gone into the design and construction of equipment for small surfaces, which is to be welcomed only if it provides a functional and economic solution to the specific problem of the farmer. Under that approach, the objective of this study was to design, build and evaluate a low-cost horse drawn seeder, eventually human, using materials that are easy to acquire.

A prototype was designed and built using parts and components made of iron plates, wood, bicycle pinions and chains with promising results in both static and dynamic tests, using a disk of 16 holes that locates 6.8 seeds per meter with a coefficient of variation of $4.5 \%$ with respect to the expected value, with $85.4 \%$ of the seeds well placed, without loss by damage to the seeds. Additionally, a seeding depth of $4.72 \mathrm{~cm}$ was achieved, against an expected depth of $5 \mathrm{~cm}$.

\section{RESUMEN}

La mecanización resulta generalmente difícil para los pequeños agricultores ya que no se encuentra accesible por motivos de costo, o bien, no se adapta a sus necesidades. Se han hecho muchos esfuerzos sobre diseño y construcción de equipos para pequeñas superficies, pero éstos serán acogidos, sólo si se entrega una solución funcional y económica a un problema puntual del agricultor. Bajo ese criterio, se procedió a realizar el presente trabajo, con el objeto de diseñar, construir y posteriormente evaluar una unidad sembradora de tiro animal y eventualmente humano de bajo costo, construido con materiales de fácil adquisición.

Se diseñó y construyó un prototipo con partes y componentes de pletina de hierro; madera; piñones y cadenas de bicicleta con promisorios resultados, pudiendo obtener 5 relaciones de transmisión y un disco dosificador de fácil fabricación, obteniéndose resultados promisorios, tanto en pruebas estáticas como dinámicas, usando un disco de 16 alvéolos que ubica 6,8 semillas por metro lineal con un coeficiente de variación de 4,5 \% respecto al valor esperado, con 85,4 \% de las semillas bien colocadas y sin pérdidas por daño ya que no se vio afectada la germinación. A su vez, se logró una profundidad de siembra de 4,72 cm, ante una profundidad esperada de $5 \mathrm{~cm}$.

Palabras clave: Tecnología intermedia, mecanización, pequeños productores, siembra, Zea mays.

\section{INTRODUCCIÓN}

La participación de la máquina en los procesos productivos desde sus inicios, ha tenido una gran influencia en el aumento del producto obtenido. La agricultura no escapa a este proceso, desde el tractor, arados, sembradoras, máquinas recolectoras, cosechadoras de plantas, etc. (Ortiz-Cañavate, 2003). Sin embargo, las nuevas tecnologías han provocado cada vez más una brecha entre los grandes productores que tienen acceso a la tecnología, y los pequeños a los que les re- sulta imposible de alcanzar, ya sea por precios o por oportunidad de uso (FAO, 1987). Según Echenique y Gómez (1988), la brecha tecnológica y las consiguientes diferenciaciones de rendimientos entre la pequeña agricultura en comparación con la mediana y grande, ha contribuido también a reducir su peso relativo en la producción sectorial. En gran medida se debe a que los productores de maquinarias no tienen desarrollado modelos de menor costo y utilizables en pequeñas superficies, necesitándose máquinas diseñadas para las distintas condiciones de los productores y no que 
los productores se ajusten a las máquinas. En algunos países en desarrollo se ofrecen alternativas como la propuesta, pero también resultan difíciles de obtener por lo que se hace necesario desarrollar prototipos locales sin necesidad de realizar engorrosas importaciones (Pachak, 1985). Por otro lado, fuera de aspectos tecnológicos y económicos, hay ciertas condiciones locales, como tamaño de la propiedad, o condiciones geográficas, que el uso de tracción animal es la única posibilidad (Arriaga et al., 2003).

En cultivos como el maíz, los pequeños productores comparados con los medianos, pueden llegar a presentar rendimientos menores en porcentajes cercanos al 36\% de lo que deberían obtener. Esto se debe principalmente a problemas en la preparación del suelo o de la siembra (Faiguenbaum, 2003), considerando que la siembra, es sin duda la operación más importante del ciclo productivo (Gracia y Palau, 1983). En el caso de llegar a usar sembradora, deben recurrir al arriendo, lo que implica poca seguridad de una siembra oportuna y una disminución de sus ingresos. Al contar con máquinas propias y de bajo costo, se superarían estos inconvenientes, puesto que la siembra a mano no ofrece la calidad de una máquina simple. Ruíz y Riquelme (2007), mencionan que en el caso de la siembra directa, las sembradoras de tracción animal presentan ventajas sobre las sembradoras de tracción motriz, a condición que el agricultor disponga de animales y opere el mismo la sembradora, dado que en estas condiciones no le significan una salida de recursos económicos por el total de la labor de siembra.

Considerando lo discutido y analizado anteriormente, se estimó necesario desarrollar, construir y evaluar una sembradora de diseño simple, hecha con piezas y componentes que se encuentran en el mercado, de bajo costo y que llenen el vacío tecnológico antes expuesto. Por lo tanto, el objetivo del presente trabajo es:

-Diseñar, construir y evaluar una unidad sembradora monograno de bajo costo, para tracción animal y eventualmente humana.

\section{MATERIALES Y MÉTODOS}

\section{Lugar y época de trabajo}

El desarrollo de esta investigación se llevó a cabo en la Facultad de Ciencias Agronómicas de la Universidad de Chile. El prototipo fue construido en el laboratorio de Mecanización Agrícola del Departamento de Ingeniería y Suelos y las pruebas de terreno, se efectuaron en un sector de la Estación Experimental Antumapu de dicha Facultad, que corresponde cartográficamente a la serie Santiago, caracterizándose por un suelo de una textura Franco arenosa, buen drenaje y de topografía plana con una pendiente de 1,2\% (CIREN, 1999).

\section{Materiales}

El prototipo está compuesto por materiales de bajo costo de adquisición y, en lo posible, que permitiera una construcción propia por parte del usuario.

Los componentes funcionales más importantes son los siguientes: soporte o chasis, mecanismo de transmisión de fuerzas que da movimiento al sistema dosificador, tubo de descarga, abresurco y, por último, un sistema tapador - apisonador, como se muestra en la figura 1.

Este prototipo está compuesto de una estructura o chasis construido en base a pletinas metálicas, una tolva de semillas metálica de 22,4 L, que va colocada sobre un sistema dosificador de plato alveolado inclinado. El movimiento del plato alveolado proviene desde su rueda de apoyo, de $39 \mathrm{~cm}$ de diámetro en cuyo eje tiene un piñón chicharra unidireccional intercambiable de 18 dientes, el que mediante una cadena de bicicleta da movimiento al piñón más grande (44 dientes) de un piñón doble (corona de bicicleta con 44 y 16 dientes), permitiendo así una reducción de la velocidad de giro. A continuación el otro piñón de esa corona doble, es a su vez, conductor de 16 dientes de un piñón múltiple de bicicleta de cinco velocidades $(24,21,19,17$ y 14 dientes), permitiendo una regulación de las velocidades de giro (y por ende de la dosificación) (Figura 1). Este último piñón presenta un buje de technyl que se desliza sobre un eje estriado en el sentido de no producir movimiento, lo cual se logra gracias a la presencia de un embrague deslizante accionado desde uno de los brazos de las manceras. Mediante dos crucetas en el eje se logra cambiar la orientación del movimiento del plato dosificador, desde una posición horizontal y perpendicular al avance de la unidad sembradora, hasta una inclinación de unos $45^{\circ}$ y paralelo al avance. Mediante un tubo de goma baja la semilla hasta el abresurco tipo patín (el cual no es rígido y puede pivotear) y por último, un sistema tapador - apisonador termina la labor sobre el suelo (Figura 2).

\section{Método}

El desarrollo de esta máquina se inició con un diseño previo, el cual se fue modificando a medida que se fueron construyendo los distintos prototipos, probándose sus partes para lograr un funcionamiento satisfactorio con la tecnología incorporada. Para lograr este objetivo se realizaron dos formas de evaluaciones:

Evaluaciones preliminares, destinadas a ensayar a medida que se avanzaba en la construcción de los distintos componentes, perfeccionando aquellos que presentaron inconvenientes en pruebas estáticas y dinámicas y evaluaciones finales, en terreno comparando el prototipo obtenido con otras sembradoras. 


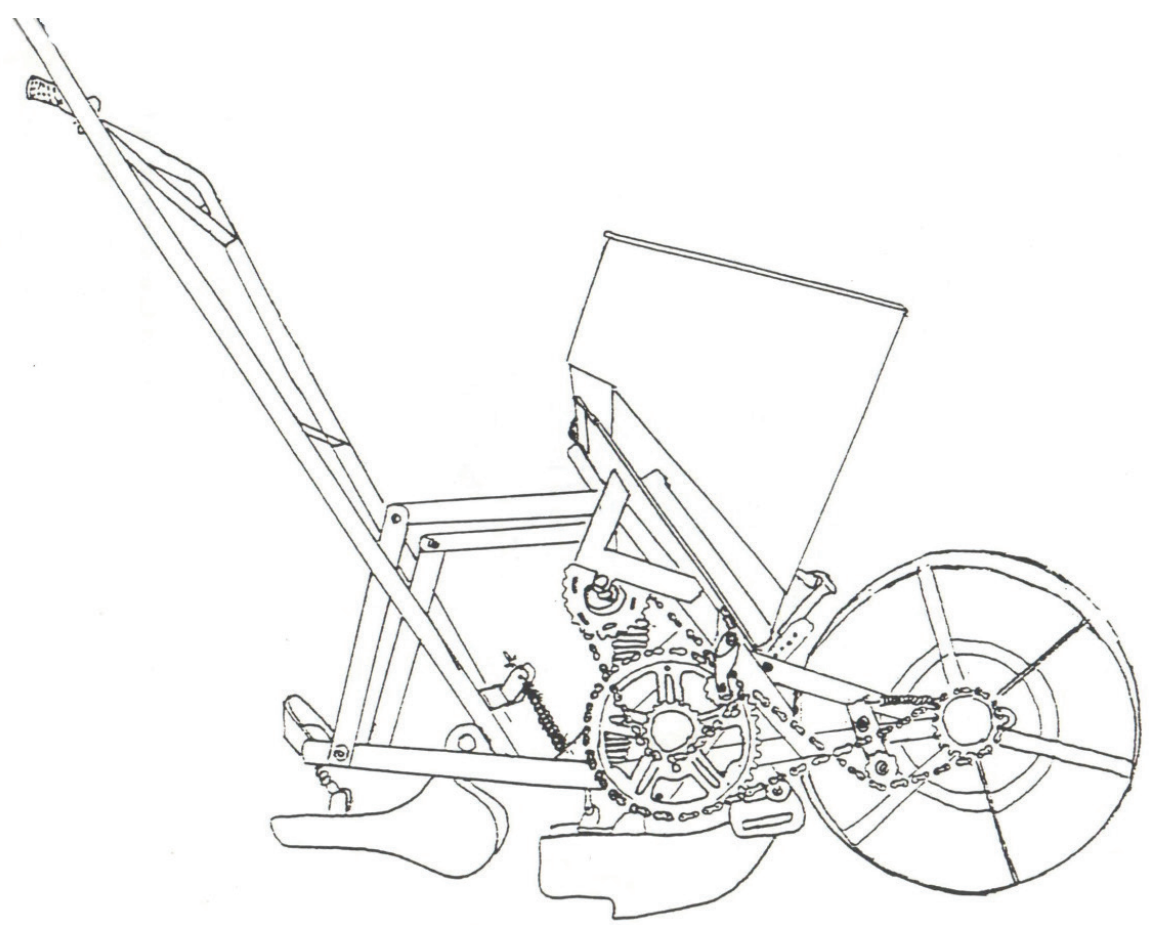

Figura 1. Esquema general del prototipo.

Figure1. General diagram of the prototype.

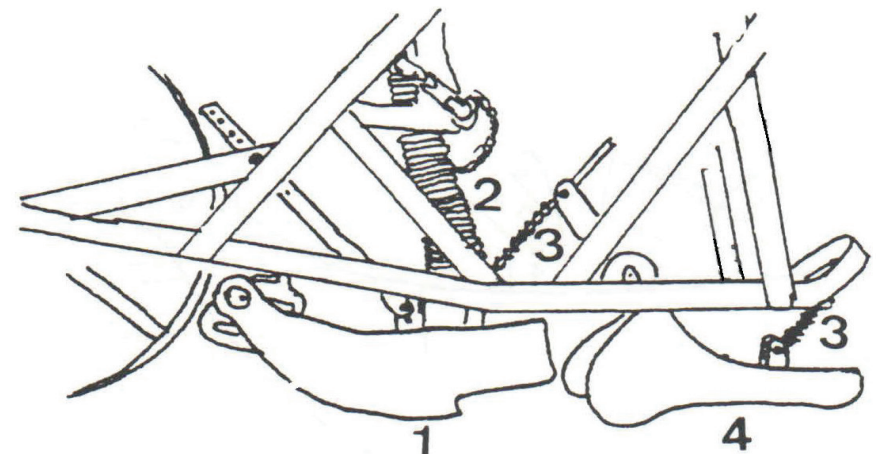

Figura 2. Detalles abresurco y mecanismo tapador apisonador. 1. Abresurco tipo patín, 2. Tubo descarga, 3. Tensores, 4. Mecanismo tapador-apisonador.

Figure 2. Details opener and crushing packer mechanism. 1 Runner-type opener, 2. Tube download, 3. Tensors, 4) Crushing packer mechanism.

\section{Evaluaciones preliminares}

En esta etapa de diseño, basado en el diseño previo, fue muy importante realizar y justificar las innovaciones que pudieran realizarse. A medida que se avanzaba en la construcción, se estudiaron y probaron las distintas partes de la máquina, perfeccionándose aquellas partes según los resultados obtenidos en las pruebas estáticas como dinámicas realizadas. Siendo el mecanismo de transmisión de movimiento y el mecanismo dosificador los más importantes de una sembradora, se procedió de la siguiente manera:

Mecanismo de transmisión de fuerza: Este proporciona el movimiento sincronizado al dosificador, determinando el espaciamiento de las semillas en el surco. Está formado por una rueda, la que al girar transmite movimiento mediante una combinación de piñones intercambiables a un disco dosificador, movimiento que se conoce como índice de transmisión (" $i$ "), que es igual a: 


\section{$\frac{\text { Producto engranajes conductores }(Z \text { conductores })}{\text { Producto engranajes conducidos }(Z \text { conducidos })}=$}

Lo que entrega el número de vueltas del disco dosificador, por cada vuelta de la rueda.

Disco dosificador: Se probaron distintos diseños de dientes y de alvéolos. En forma estática se hizo conteo de semillas girando la rueda 10 veces, con una apreciación óptica del llenado de los alvéolos o de los dientes, según sea el caso, a una velocidad tangencial (periférica) del disco que simulase una velocidad de avance de $4 \mathrm{~km} \mathrm{~h}^{-1}$.

El cálculo de la velocidad tangencial del disco se realizó según el método descrito por Delafosse (1986) y por Delafosse et al. (1987), considerando una velocidad de trabajo de $4 \mathrm{~km} \mathrm{~h}^{-1}$ según la fórmula:

$$
V_{t g}=\frac{V_{a}\left(m \cdot s^{-1}\right) \times i \times r P(m)}{r R(m)}
$$

Donde:

$V t g$ : velocidad tangencial

Va: velocidad de avance de la máquina

$i$ : índice de transmisión

$r P$ : radio de la placa de siembra centro del alvéolo

$r R$ : radio de la rueda de mando bajo carga

En forma dinámica se observó el espaciamiento de las semillas al hacer funcionar el prototipo sobre cemento y sobre pasto para evitar el rebote de las semillas.

Luego, en terreno, se observó el correcto funcionamiento de los mecanismos de tubo de descarga, abresurco y sistema tapador-apisonador, realizándole los ajustes necesarios para evitar rebote, y que la semilla quede bien posicionada y tapada.

\section{Evaluaciones finales}

La unidad sembradora prototipo se evalúo en terreno mediante su comparación con dos tipos de siembra de uso común en la agricultura, una mecánica de tarro y otra con un bastón sembrador.

Se realizaron ensayos de campo, sembrándose parcelas de cuatro hileras con cada uno de los sistemas de siembra antes mencionados, en un terreno previamente preparado en forma óptima, usándose semilla desinfectada y realizando una aplicación de Basudin $10 \mathrm{G}$ contra gusanos cortadores, suministrado después de la siembra incorporado mediante el uso de ramas, para así aislar el aspecto "máquina" del experimento. $\mathrm{Al}$ mismo tiempo se tomaron muestras de suelo para la determinación del contenido de humedad mediante el secado de las muestras a $105 \mathrm{C}^{\circ}$ por 72 horas (Sandoval et al., 2012).
Las parcelas tuvieron un tamaño de aproximadamente 15,65 metros de largo por 3,5 metros de ancho, sembrándose 4 hileras en cada una de ellas a $75 \mathrm{~cm}$ de distancia y una densidad de siembra lo más cercana a 6,15 plantas por metro, con una profundidad de $5,0 \mathrm{~cm}$, siendo esta la máxima profundidad recomendada por Bragachini, et al. (2002). Con éstos antecedentes se evaluó la regularidad de la siembra, según el rango de espaciamiento de las semillas, considerándose la normativa existente en Argentina y mencionada por Delafosse (1986), donde se considera como falla de siembra distancias iguales o superiores al $50 \%$ más que lo teórico, y a su vez como multiplicidad de siembra distancias menores o iguales al 50\% del espaciamiento teórico.

En forma complementaria al ensayo se midieron otros dos parámetros:

a) Profundidad de siembra: luego de realizarse el conteo y la medición de la distancia entre plantas, se midió la profundidad de las semillas.

b) Daño mecánico: se recolectaron muestras de semillas que hayan pasado por cada una de las sembradoras y una muestra testigo sacada desde la bolsa de semillas, para ver el posible daño mecánico que pudo haber ocurrido, lo que se realizó mediante una inspección visual y un test de germinación realizado en cámara de germinación a $25 \mathrm{C}^{\circ}$ por 7 días.

Al alcanzar las plantas una altura aproximada de $10 \mathrm{~cm}$, se procedió al conteo del número de plantas existentes en tres tramos de 1 metro cada uno en las dos hileras centrales, para así determinar la población lograda.

\section{Diseño experimental y tratamientos}

El ensayo se dispuso bajo el diseño de bloques completos al azar con cinco bloques y tres tratamientos, donde cada tratamiento correspondió a una máquina distinta. La unidad experimental fue una parcela de 55 $\mathrm{m}^{2}$.

Cada parcela correspondió a una pasada de cada máquina (cuatro hileras) y en el caso de la unidad sembradora para tracción animal y del bastón sembrador se realizaron cuatro pasadas.

Siendo los tratamientos:

Tratamiento 1: Bastón sembrador Tratamiento 2: Sembradora de tarro Tratamiento 3: Prototipo

Como los valores obtenidos, al considerarlos como promedios, no son comparables unos de otros, ya que cada máquina está ajustada para dar una cierta dosificación que no será la misma entre ellas, se procedió a 
compararlos mediante la construcción de un índice relativo expresado en porcentaje, que mide la desviación de los valores obtenidos respecto de los esperados. La fórmula usada fue:

$\%$ variacion con respecto al valor esperado $=\frac{\mid \text { Valor obtenido }- \text { Valor esperado } \mid}{\text { Valor esperado }} \times 100$

Además, se determinó el Coeficiente de variación, para comparar la dispersión entre los tratamientos.

Los datos expresados en porcentaje fueron transformados en Grados Bliss, para normalizar los valores de los tratamientos, y permitir su comparación a través de análisis de varianza.

En el test de germinación se realizó un diseño completamente al azar con cuatro tratamientos y cuatro repeticiones cada uno, correspondiendo tres tratamientos con semillas que pasaron por cada sembradora y un cuarto tratamiento $\left(\mathrm{T}_{0}\right)$ con semillas que no pasaron por las máquinas sembradoras.

La unidad experimental del test de germinación correspondió a grupos de 50 semillas.

\section{Análisis estadístico.}

Con el propósito de comparar las distintas máquinas sembradoras, a los resultados se les aplicó análisis de varianza (ANDEVA). Cuando se encontraron diferencias significativas entre los tratamientos, se usó la prueba de comparaciones múltiples de Duncan $(\alpha=$ $0,05)$.

\section{RESULTADOS Y DISCUSIÓN}

\section{Resultados de los ensayos preliminares}

Los resultados de los ensayos preliminares permitieron llegar a un prototipo final (figura 3), el cual tiene una masa de 32 kilos, una capacidad de la tolva de 22 litros y un tamaño reducido, el cual permite ser replicado con facilidad, con un costo de materiales de 103 USD, y que presenta las siguientes partes:

\section{Sistema de transmisión}

La implementación de un sistema intermedio de reducción de revoluciones, permitió que el giro del plato dosificador fuese más lento, y el empleo de un piñón múltiple, logró que se pudiese tener 5 alternativas de giro, y por ende ante un mismo plato dosificador, 5 dosis diferentes de semillas, mostrándose en el Cuadro 1 , los respectivos índices de transmisión.

\section{Mecanismo dosificador de disco}

Se ensayaron diferentes posibilidades de discos, ya sea construidos temporalmente con fibra de densidad media (MDF), adaptación de discos de una sembradora John Deere modelo 24B, o bien de materiales especiales como Durocoton, el cual ofrecía las garantías de durabilidad, manteniéndose el uso de MDF a lo largo de los ensayos para probar distintas posibilidades. Así, se vio que a pesar del trabajo al cual fue sometido, el MDF es comparable a los demás materiales en cuanto a su

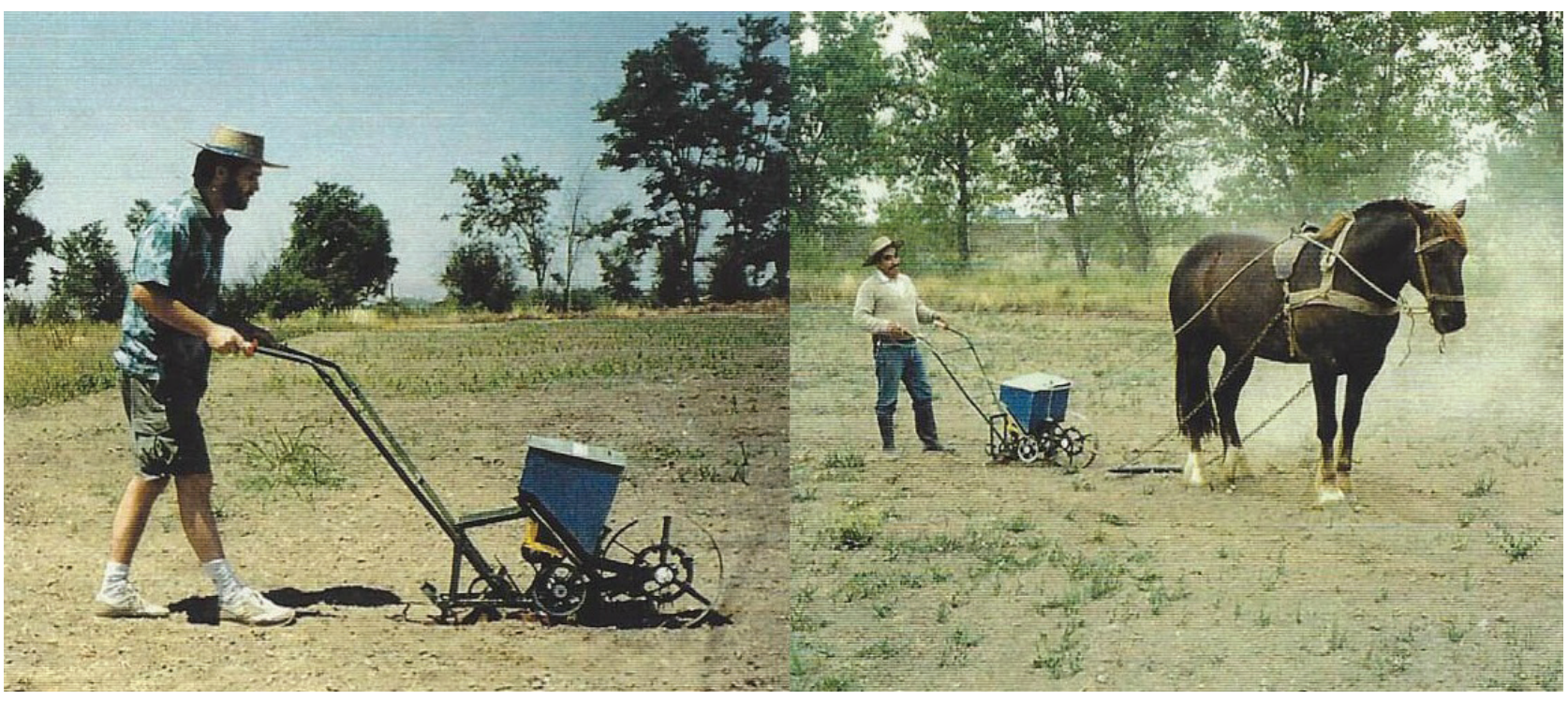

Figura 3. Prototipo en terreno mediante tracción humana (izquierda) y tracción animal (derecha).

Figure 3. Prototype in field: human traction (left) and animal traction (right). 
resistencia, decidiéndose seguir usando este material ya que su costo es mucho más bajo (50 veces), es un material de fácil acceso y se puede construir el disco dosificador sin necesidad de elementos especiales, debiéndose tener la precaución que el tamaño del alveolo perforado, sea $10 \%$ más grande que semilla de mayor tamaño (Agnes et al., 2010).

La velocidad tangencial de desplazamiento del alvéolo como su índice de transmisión se aprecia en el Cuadro 1.

Estos valores son bastante inferior a lo máximo de desplazamiento descrito por Delafosse (1986) y Delafosse et al., (1987) de 0,315 m.s. (ASAE) y 0,29 m.s. (FAO).

Esto permitiría poder realizar la siembra a una mayor velocidad, pero a su vez, este teórico aumento en la velocidad, podría afectar otros parámetros como el tiempo disponible de la semilla para salir del alvéolo lo que provocaría que las semillas cayeran más seguidas, aumentando el riesgo de provocar un distanciamiento excesivo o una duplicidad de siembra por efecto del rebote de las semillas al interior del tubo de descarga.

\section{Prueba de campo y resultados}

Se procedió a realizar una siembra en el campo, eligiéndose una población de aproximadamente 81.000

Cuadro 1. Velocidades periféricas según piñón múltiple elegido (distintos índices).

Table 1. Peripheral speeds depending on chosen multiple sprocket (various indices).

\begin{tabular}{ccc}
\hline $\begin{array}{c}\mathbf{N}^{\circ} \text { dientes } \\
\text { piñon }\end{array}$ & $\begin{array}{c}\text { Índice de } \\
\text { transmisión }\end{array}$ & $\begin{array}{c}\mathbf{V t g} \\
\left(\mathbf{m ~ s} \mathbf{~ s}^{-1}\right)\end{array}$ \\
\hline 14 & 0,4680 & 0,234 \\
17 & 0,3850 & 0,193 \\
19 & 0,3445 & 0,173 \\
21 & 0,3117 & 0,156 \\
24 & 0,2727 & 0,137 \\
\hline
\end{tabular}

plantas por hectárea, valor que fue elegido debido a que la sembradora John Deere (Modelo 24B) no contaba con una amplia gama de piñones y de discos alveolados. La única opción compatible con las posibilidades del prototipo, fue elegir una combinación de disco de 16 alvéolos con el piñón múltiple de 14 dientes.

Para el caso del bastón sembrador, se definió que debe sembrarse usando la marca de $16 \mathrm{~cm}$, para así lograr una población estimada de 83.250 plantas por hectárea, plantados dos semillas por vez.

\section{Evaluación de la población obtenida}

En el caso del número de plantas por metro lineal (Cuadro 2), se observó que no se advirtieron diferencias significativas entre los tratamientos $\mathrm{T}_{1}$ y $\mathrm{T}_{3}$ pero no así con $\mathrm{T}_{2}$ al analizar el porcentaje de variación con respecto a la cantidad de plantas esperadas por metro lineal, que indican que mientras más pequeño es ese valor, mejor es el resultado.

Al considerar el Coeficiente de Variación (CV), o sea, al relacionar la desviación estándar (DS) con los promedios, no se encontraron diferencias significativas entre los tratamientos.

En la Figura 4, se muestra la distribución del número de plantas por metro en el momento de la emergencia para el prototipo, apreciándose que los valores no se alejan mucho de lo esperado $(6,1$ plantas por metro), obteniéndose en promedio 6,8 plantas.

Al considerar la distancia entre las plantas y/o semillas en un metro lineal, se observa en el cuadro 3 que se obtiene el mejor resultado con el prototipo, al considerar el porcentaje de variación con respecto a la distancia esperada, seguido de $\mathrm{T}_{1}$, $\mathrm{y}$ por último el $\mathrm{T}_{3}$ el cual presenta la mayor variación.

Los resultados del cuadro 3 pueden ser un poco engañosos, ya que en terreno se observó claramente que $\mathrm{T}_{1}$ (bastón sembrador), presentaba mejor distribución de las plantas con un promedio más alto pero los valores estaban más cercanos, mientras que el prototipo $\left(\mathrm{T}_{3}\right.$ ) presentaba una amplia gama de distancias (alta DS), lo que ayudó a lograr un mejor promedio.

Cuadro 2. Número promedio de plantas en un metro lineal

Table 2. Average number of plants in a linear meter

\begin{tabular}{|c|c|c|c|c|c|}
\hline \multirow{2}{*}{ Tratamiento } & & \multicolumn{2}{|l|}{ Numero plantas } & \multirow{2}{*}{$\begin{array}{l}\text { Porcentaje variación } \\
\text { respecto esperado (\%) }\end{array}$} & \multirow{2}{*}{$\begin{array}{l}\text { Coeficiente } \\
\text { de variación }\end{array}$} \\
\hline & & promedio & esperadas & & \\
\hline Bastón sembrador & $\mathrm{T}_{1}$ & 5,57 & 6,25 & 11,96 a & $12,61 \mathrm{a}$ \\
\hline Sembradora mecánica & $\mathrm{T}_{2}$ & 8,60 & 6,10 & $40,62 \quad b$ & 15,19 a \\
\hline Prototipo & $\mathrm{T}_{3}$ & 6,80 & 6,11 & $4,47 a$ & 14,17 a \\
\hline
\end{tabular}

* valores seguidos por letras distintas presentan diferencias significativas al 0,05 


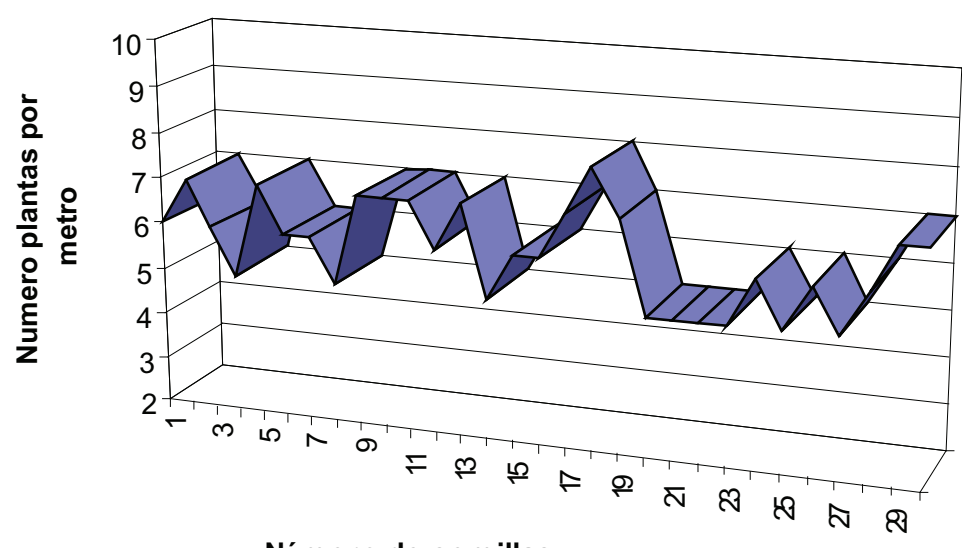

Número de semillas

Figura 4. Distribución del número de plantas por metro del prototipo.

Figure 4. Distribution of the number of plants per meter of the prototype.

Cuadro 3. Distancia promedio (cm), entre plantas y/o semillas en un metro lineal.

Table 3. Average distance $(\mathrm{cm})$ between plants or seeds in a linear meter.

\begin{tabular}{|c|c|c|c|c|c|}
\hline \multirow{2}{*}{ Tratamiento } & & \multicolumn{2}{|c|}{ Distancia entre plantas $(\mathrm{cm})$} & \multirow{2}{*}{$\begin{array}{l}\text { Porcentaje variación } \\
\text { respecto esperado (\%) }\end{array}$} & \multirow{2}{*}{$\begin{array}{l}\text { Coeficiente } \\
\text { de variación }\end{array}$} \\
\hline & & promedio & esperadas & & \\
\hline Bastón sembrador & $\mathrm{T}_{1}$ & 18,06 & 16,00 & $16,06 \mathrm{~b}$ & 20,41 a \\
\hline Sembradora mecánica & $\mathrm{T}_{2}$ & 8,10 & 16,35 & $47,79 \quad \mathrm{c}$ & 67,13 \\
\hline Prototipo & $\mathrm{T}_{3}$ & 15,20 & 16,38 & $6,44 \mathrm{a}$ & 44,18 b \\
\hline
\end{tabular}

* valores seguidos por letras distintas presentan diferencias significativas al 0,05

Este fenómeno se observa al examinar el Coeficiente de Variación (Cuadro 3), en donde los papeles se invierten, siendo ahora $\mathrm{T}_{1}$ mejor que $\mathrm{T}_{3}$, mayormente dado por la influencia de la alta DS que presenta $T_{3}$ permaneciendo $\mathrm{T}_{2}$ como el que presenta peores características. De todas maneras, para los tres tratamientos el CV es alto si se considera que un valor sobre 20 es excesivo $^{1}$.

Delafosse (1986) y Delafosse et al. (1987), señalan que la desviación estándar (considerando la distancia entre plantas) muestra el grado de precisión de una máquina sembradora, por lo cual si consideramos este parámetro, el prototipo aunque presentó los mejores resultados, no lo fue en su grado de precisión.

Esta menor precisión, considerando la distribución de la semilla, se debe probablemente a la altura de caída de ella desde el dosificador hasta el fondo del surco, ya que mientras mayor es la altura, existe más posibilidades de fallas de siembra, pues las semillas, al caer, pueden tener distintas trayectorias (derecho,

Fernandez, C. Ing. Agr. Depto. Ing. y Suelos. Fac. Cs. Agronómicas. Universidad de Chile. (Comunicación personal). con rebotes, etc.), lo que motivará distintos tiempos de caída y, por ende distintos espaciamientos. Al respecto Brecce et al. (1975), mencionan que a una velocidad de $5 \mathrm{~km} \mathrm{~h}^{-1}$ con un espaciamiento de $5 \mathrm{~cm}$, si una semilla se retrasa $1 / 30$ segundos al caer, será alcanzada por la semilla siguiente; otro motivo, es el leve juego que presentan las crucetas, lo que motiva que se traduzca en cambios en la velocidad de giro del dosificador, problemas motivados solamente por falta de mayor presión al colocar los remaches.

En el cuadro 4, se presenta la frecuencia de los rangos de espaciamiento de las semillas sembradas por el prototipo, encontrándose un $85,4 \%$ de semillas bien colocadas, valor que es considerado deficiente, pero es bastante alto para la tecnología y los medios empleados. Con respecto a lo anterior, Delafosse (1986), señala que el mínimo valor permitido debe ser $90 \%$, sin embargo Ossandon ${ }^{2}$ señala que para esta tecnología basta con un $80 \%$. Las semillas mal colocadas, ya sea por multiplicidad como por fallas se pueden deber a la precisión de siembra, mencionada anteriormente.

\footnotetext{
Ossandón, E. Ing. Agr. (Comunicación personal).
} 
Cuadro 4. Frecuencia de rangos de espaciamiento en el prototipo.

Table 4. Frequency ranges of spacing in the prototype.

\begin{tabular}{lccc}
\hline & Rango (cm) & Casos & Porcentaje \\
\hline $\begin{array}{l}\text { Multiplicidad de } \\
\text { siembra }\end{array}$ & $0,0-8,19$ & 18 & 10,98 \\
Semilla unitaria & $8,20-24,56$ & 140 & 85,37 \\
\hline Fallas de siembra & $\geq 24,57$ & 6 & 3,66 \\
\hline
\end{tabular}

Los valores, tanto en número de semillas como en la distancia, en $\mathrm{T}_{1}$ están levemente sobre valorados, ya que se sembraron dos semillas por golpe, encontrándose en algunos casos una sola semilla, y en otros más de dos, aceptándose en ambas circunstancias como semilla bien colocada.

\section{Evaluación de la profundidad de siembra}

El prototipo $\left(\mathrm{T}_{3}\right)$ presenta claramente una mejor profundidad de siembra ya que es la más cercana a la esperada $(5,0 \mathrm{~cm})$, a su vez el sistema tapador - apisonador logra hacer un pequeño camellón que permite, por lo menos, en aquellas semillas que por algún motivo hubiesen quedado superficiales dejarlas cubiertas con unos $2 \mathrm{~cm}$ de suelo lo que les permitió germinar.

No ocurrió lo mismo para los otros tratamientos, en donde la profundidad del bastón sembrador $\left(T_{1}\right)$ fue baja, debido que ella está dado, por la fuerza con que el operador presiona el bastón, parámetro claramente determinado por el cansancio que afecte al operador, como también por la presencia de raíces, terrones y otros elementos que obstruyan el paso del bastón, aunque no afectó a la semilla utilizada, debido a que requiere poca profundidad. El tratamiento con la máquina sembradora mecánica, presentó un promedio de profundidad bajo, debido al alto número de semillas descubiertas (Cuadro 5).

En la Figura 5 se comparan los dos mejores tratamientos, ambos con una profundidad esperada de $5 \mathrm{~cm}$.

A diferencia del punto anterior, en el caso de la sembradora de tarro, aunque también se obtuvo un promedio bajo, presentó una gama más amplia de profundidades, desde semillas descubiertas, que fueron comidas por las aves, hasta semillas muy profundas que no germinaron. Esto, debido a que la sembradora no logró adaptarse a las marcadas irregularidades del terreno.

En el caso del prototipo las irregularidades del terreno lograron ser sorteadas, ya que al trabajar libremente, y no montado en una barra porta herramienta, se adaptó mejor a los cambios del terreno y, a su vez, el operador puede a través de las manceras acomodar
Cuadro 5. Profundidad promedio de los distintos tratamientos, con un valor esperado de $5 \mathrm{~cm}$.

Table 5. Average depth of the different treatments, with an expected value of $5 \mathrm{~cm}$.

\begin{tabular}{lccc}
\hline \multirow{2}{*}{ Tratamiento } & \multicolumn{2}{c}{$\begin{array}{c}\text { Profundidad de las } \\
\text { semillas }(\mathrm{cm})\end{array}$} \\
\cline { 2 - 4 } & & Promedio & Esperado \\
\hline Bastón sembrador & $\mathrm{T}_{1}$ & $3,59 \mathrm{~b}$ & 5,00 \\
Sembradora mecánica & $\mathrm{T}_{2}$ & $3,51 \mathrm{~b}$ & 5,00 \\
Prototipo & $\mathbf{T}_{\mathbf{3}}$ & $\mathbf{4 , 7 2} \mathbf{~ a}$ & $\mathbf{5 , 0 0}$ \\
\hline
\end{tabular}

* valores seguidos por letras distintas presentan diferencias significativas al 0,05

la máquina, ya sea subiendo un poco la parte posterior al haber una elevación, o bien presionar para hundir la parte posterior al existir una depresión.

En general, se observó que el prototipo presentó una adecuada profundidad y tapado de semillas, aspecto importante que suele ser problemático en sembradoras de tracción animal (Bogado et al., 2006).

\section{Evaluación del daño producido a las semillas}

Posteriormente a la siembra se procedió a determinar el daño producido en las semillas, mediante una inspección visual de ellas y un test de germinación. Además, se realizó un tratamiento testigo $\left(\mathrm{T}_{0}\right)$ con semilla que no había pasado por ninguna de las sembradoras, resultados que se pueden apreciar en el cuadro 6 .

El mayor daño en las semillas se presentaba en ellas antes del ensayo y no por motivos mecánicos de los distintos tratamientos (a excepción de $\mathrm{T}_{2}$ ). Esto se aprecia por las semillas dañadas en el tratamiento testigo $\left(\mathrm{T}_{0}\right)$, donde eran semillas sacadas directamente de la bolsa y no pasaron por ningún mecanismo dosificador (presentando daño recubierto con la aplicación de fungicida e insecticida), mientras que en $\mathrm{T}_{2}$ se presentaron granos partidos producidos por la máquina.

En el caso del test de germinación las tendencias son semejantes a las vistas previamente en el caso de los daños mecánicos. En el cuadro 6, se puede apreciar que no existen diferencias significativas con respecto al testigo, aunque el prototipo y el bastón sembrador presentaron valores más cercanos, mientras que $\mathrm{T}_{2}$ mostró una disminución en su valor de germinación. Además, se puede apreciar un leve aumento en la germinación del $\mathrm{T}_{3}$, con respecto al testigo, fenómeno también visto por estudios realizados por Delafosse (1986), en donde al ensayar tres distintos sistemas de dosificación en sembradoras mecánicas observó un leve incremento del poder germinativo con respecto al testigo. 


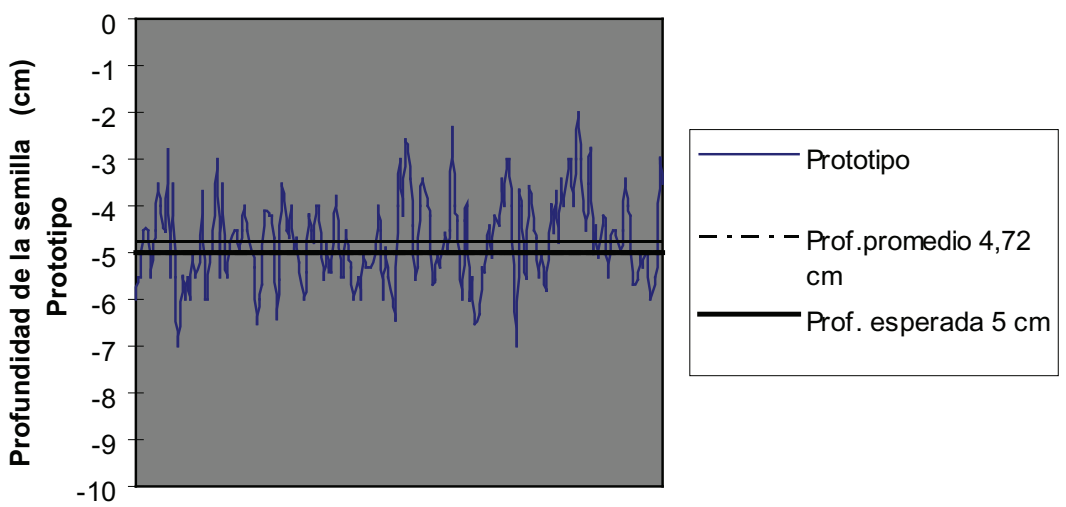

Figura 5. Profundidades obtenidas usando el prototipo y el "bastón sembrador" en centímetros.

Figure 5. Depths obtained using the prototype and the "sower cane" in centimeters.

Cuadro 6. Número de semillas dañadas y germinadas de un total de 50 en cada repetición.

Table 6. Number of damaged and germinated seeds from a total of 50 in each repetition.

\begin{tabular}{|c|c|c|c|c|c|}
\hline \multicolumn{2}{|l|}{ Tratamiento } & \multicolumn{2}{|c|}{ Semillas dañadas } & \multicolumn{2}{|c|}{ Semillas germinadas } \\
\hline & & promedio & $\%$ & promedio & $\%$ \\
\hline Testigo & $\mathrm{T}_{0}$ & 1,75 & $3,44 \quad b$ & 165 & $85,61 \mathrm{ab}$ \\
\hline Bastón sembrador & $\mathrm{T}_{1}$ & 2,00 & $4,00 \quad b$ & 163 & 83,65 a \\
\hline Sembradora mecánica & $\mathrm{T}_{2}$ & 4,25 & 8,48 & 152 & 79,91 a \\
\hline Prototipo & $\mathrm{T}_{3}$ & 0,75 & 1,13 a & 176 & 89,55 b \\
\hline
\end{tabular}

* valores seguidos por letras distintas presentan diferencias significativas al 0,05

\section{CONCLUSIONES}

Considerando los datos obtenidos en las evaluaciones, así como las experiencias que se obtuvieron durante el desarrollo de esta investigación se puede concluir que: en general, el prototipo cumplió las expectativas que se tenían sobre él, desde el aspecto económico como del funcional. Se logra obtener una buena siembra, donde comparado con los otros dos equipos, presento el menor daño de semillas y la más alta germinación; la profundidad más correcta y el mejor número de plantas por metro lineal, aunque existió una cierta irregularidad en las distancias de siembras, atribuibles a la altura de caída de las semillas, aspecto solucionable mediante la disminución de dicha altura. El prototipo no presenta inconvenientes técnicos para poder ser construido por pequeños productores, ya que se diseñaron y utilizaron piezas sencillas y relativamente fáciles de hacer o adquirir y a un bajo costo.

\section{REFERENCIAS}

Agnes, D., Tourn, M., Soza, E., 2010. Variabilidad en la distribución de semillas de maíz: una revisión. Revista de la Facultad de Agronomía 30(1-2), 101-121.

Arriaga, C., Castelán, O., Velázquez, L., 2003. Investigación en animales de trabajo para el desarrollo rural. Universidad Autonoma del Estadio de Mexico (UAEMEX), Toluca.

Bogado, H., Paredes, F., Esparza, S., 2006. Principales problemas en sembradoras no tractorizadas para siembra directa, para la pequeña agricultura familiar en la provincia de corrientes, Argentina, in: Díaz-Rossello, R., Rava, C. (Eds.), Aportes de la ciencia y la tecnología al manejo productivo y sustentable de los suelos del cono sur: IICA, PROCISUR, Montevideo, Uruguay, pp. 233-238.

Bragachini, M., Von Martini, A., Méndez, A., Pacioni, F., Alfaro, M., 2002. Siembra de maíz, eficiencia de implantación y su efecto sobre la producción de grano. Tercer Taller Internacional de Agricultura de Precisión del Cono Sur de América (PROCISUR). 17-19 de diciembre de 2002, Carlos Paz, Córdoba, Argentina, pp. 1-9. 
Breece, H., Hansen, H., Horner, T., 1975. Fundamentos de funcionamiento de maquinaria: siembra. Publicación de servicio John Deere, Illinois.

Centro de Información de Recursos Naturales (CIREN), 1999. Descripciones de Suelos y Materiales y Símbolos. Estudio Agrológico de la Región Metropolitana. Centro Información Recursos Naturales. Publicación CIREN Nº 115, Santiago.

Delafosse, R., 1986. Máquinas sembradoras de grano grueso, descripción y uso. FAO, Santiago.

Delafosse, R., Scasso, G., Melendez, C., Agurre, F., Marotto, E., 1987. La velocidad de trabajo en máquinas sembradoras de dosificación mecánica y su relación con la calidad de planteo y la eficiencia de llenado de alvéolos. Agro Ciencia 3(1), 49-57.

Echeñique, J., Gómez, S., 1988. La agricultura chilena; las dos caras de la modernización. FLACSO-AGRARIA, Santiago.

Faiguenbaum, H., 2003. Labranza, siembra y producción de los principales cultivos de Chile. Facultad de Ciencias Agrarias y Forestales, Universidad de Chile, Santiago.

Gracia, C., Palau, E., 1983. Mecanización de los cultivos hortí- colas. Mundi-prensa, Madrid.

Organización de las Naciones Unidas para la Alimentación y la Agricultura (FAO), 1987. Generación de tecnologías adecuadas al desarrollo rural. $2^{\text {a }}$ Ed. FAO, Serie Desarrollo Rural №4, Santiago.

Ortiz-Cañavate, J., 2003. Máquinas agrícolas y su aplicación. $6^{\text {ta }}$ Ed. Mundi-Prensa, Madrid.

Pachak, B.S., 1985. Selección y empleo de equipos de tracción animal, in: Food and Agricultural Organization (Eds.), Energía animal en la agricultura de África y Asia. Estudio FAO Producción y Sanidad Animal, Roma, pp. 70-83.

Ruiz, C., Riquelme, J., 2006. Sembradoras de cero labranza de tracción animal, problemas y soluciones en Chile, in: Díaz-Rossello, R., Rava, C. (Eds.), Aportes de la ciencia y la tecnología al manejo productivo y sustentable de los suelos del cono sur: IICA, PROCISUR, Montevideo, Uruguay, pp. 243-250.

Sandoval M., Dörner J., Seguel O., Cuevas J., Rivera D., 2012. Métodos de Análisis Físicos de Suelos. Departamento de Suelos y Recursos Naturales Universidad de Concepción. Publicación $N^{\circ} 5$, Chillán, Chile. 